\title{
Small bowel necrosis and enterocutaneous fistulae resulting from iatrogenic spillage of dermoid cyst contents at the time of laparoscopic surgery
}

\author{
T. Majmudar $\cdot$ H. Abdel-Rahman
}

Received: 4 November 2008 / Accepted: 10 November 2008/Published online: 2 December 2008

(C) Springer-Verlag 2008

\begin{abstract}
A 38-year-old lady with a $14 \times 8 \times 13$-cm dermoid cyst underwent a laparoscopic left salpingo-oophrectomy. Intra-operatively, there was accidental spillage of the cyst contents into the peritoneal cavity. The spilled contents were completely sucked out and a thorough peritoneal lavage was done to decrease the risk of chemical peritonitis. The patient developed disseminated granulomatous chemical peritonitis, small bowel necrosis, multiple enterocutaneous fistulae, intra-abdominal, and anterior abdominal wall abscesses. Following four further surgeries, she finally made a recovery 6 months later. Chemical peritonitis and intraabdominal abscesses are rare complications. Bowel necrosis and enterocutaneous fistulae have not been described. This case report will increase awareness of these rare complications and in their prevention and management. A literature review of strategies to prevent spillage of dermoid cyst contents at surgery is also presented.
\end{abstract}

Keywords Dermoid cyst · Benign cystic teratoma . Iatrogenic spillage · Chemical peritonitis · Entero-cutaneous fistulae

\section{Case report}

A 38-year-old lady presented to the gynecology clinic with a history of menorrhagia and left-sided lower abdominal

T. Majmudar $(\bowtie) \cdot$ H. Abdel-Rahman

Department of Obstetrics and Gynaecology,

Hinchingbrooke Hospital,

Hinchingbrooke Park,

Huntingdon, Cambridgeshire PE29 6NT, UK

e-mail: tarangmajmudar@yahoo.co.uk pain. Examination revealed a $15-\mathrm{cm}$ pelvic mass. Ultrasound scan of the pelvis reported a left adnexal cystic mass measuring $14 \times 8.1 \times 13 \mathrm{~cm}$ with a homogenous soft tissue echotexture and a small hypoechoic area. No color flow was demonstrated. The right ovary and uterus appeared normal. There was no evidence of ascites. Liver and kidneys appeared normal. Ca-125 level was $30.6 \mathrm{U} / \mathrm{ml}$. The Risk of Malignancy Index (RMI) using a scoring system suggested by the RCOG (Royal College of Obstetricians and Gynaecologists) was 91.2.

She underwent laparoscopic left salpingo-oophrectomy. At laparoscopy, a 14-cm left dermoid cyst was identified adherent to the pelvic side wall. During dissection, there was accidental rupture of the cyst causing spillage of cyst contents into the peritoneal cavity. The spilled contents were sucked out immediately and a thorough peritoneal washout performed using 51 of saline. Patient was well post-operatively, and after a 24-h observation period, discharged home.

Fifteen days after the surgery, the patient was readmitted under a general surgeon with paralytic ileus and peritonitis. $\mathrm{CT}$ abdomen revealed dilated bowel loops and free fluid in peritoneal cavity. An exploratory laparotomy was performed which confirmed granulomatous peritonitis. The abdominal cavity and bowel surfaces were covered with a white cheesy material causing small bowel adhesions. A peritoneal washout was done and an intra-peritoneal drain left in situ. The patient subsequently developed septicemia and a CT scan reported multiple intra-abdominal abscesses, the largest of which was aspirated under ultrasound guidance. The patient continued to remain septic not responding to various antibiotic regimes. A White Cell Scan revealed an abscess in the anterior abdominal wall communicating with an intra-abdominal collection. The laparotomy wound was explored. The abscess was drained 
and the wound debrided. Over the next few days, the patient redeveloped another abdominal wall abscess which was initially drained via ultrasound guidance and then via open surgery.

Twelve days later, there was dehiscence of the lower part of the laparotomy wound which discharged small bowel contents. The patient had developed a high output enterocutaneous fistula which was confirmed by a CT scan. In the following 2 weeks, the patient made recovery with conservative management. Fistula output reduced to $100 \mathrm{ml} /$ day. She was tolerating oral diet and opening her bowels. Total Parenteral Nutrition (TPN) was discontinued. She had fewer and lower spikes in temperature. She was discharged home.

At review the following week, her condition had deteriorated. She had lost $2 \mathrm{~kg}$ in weight and the fistula output had increased to $350 \mathrm{ml} /$ day. She was readmitted and naso-gastric feeding commenced. Over the next few days, the fistula output increased significantly to more than $1,500 \mathrm{ml} /$ day. CT scan revealed that the fistula had increased in size. A fistulogram revealed two other fistulae distal to the original fistula site.

The patient was transferred to a tertiary level Intestinal Failure Unit. She remained an inpatient for 2 weeks during which her nutritional status was optimized. She underwent a final laparotomy 3 months later at which dense intra-abdominal adhesions associated with disseminated granulomatous cystic lesions were noted. Three areas of fistulating disease as a result of small bowel necrosis were identified. The fistulae were excised and a segment of small bowel resected and anastomosed. The patient has since made a good recovery with no further complications.

\section{Discussion}

Dermoid cysts/benign cystic teratoma represent $10-15 \%$ of ovarian tumors. Eighty percent of all dermoid cysts occur during the reproductive years. They are usually asymptomatic but may present acutely due to torsion. Although they have a characteristic appearance on ultrasound, often, a diagnosis is only made intra-operatively. Tumor markers should be done to rule out ovarian malignancy if the diagnosis is not conclusive pre-operatively. Dermoid cysts may be managed expectantly if asymptomatic. Symptomatic and/or enlarging cysts will need surgery. An ovarian cystectomy should be performed in younger patients, preserving normal ovarian tissue.

Laparoscopic surgery in gynecology is now the standard for the surgical management of many conditions. For benign ovarian tumors laparoscopy is better than laparotomy in the reduction of febrile morbidity, urinary tract infection, postoperative complications, post-operative pain, inpatient stay, and pain [1].
Laparoscopic surgery for benign cystic teratomas (dermoid cysts) has been proved to be a safe and effective procedure [2, 3]. However, intra-operative spillage of dermoid cyst contents is known to cause chemical peritonitis which may further lead to more serious complications. This has been experimentally confirmed in a rabbit model [4]. Laparoscopy may be associated with increased risk of cyst rupture as compared to laparotomy $[5,6]$. As a result, there are concerns with operating on dermoid cysts laparoscopically.

Rates of spillage of dermoid cyst contents in different studies vary from $42.5 \%$ to $88 \%[5,7,8]$. Fortunately, the incidence of chemical peritonitis is low. A review of 14 studies reports an incidence of $0.2 \%$ [9]. The incidence of chemical peritonitis can be significantly reduced by adopting some of the following strategies at the time of laparoscopic surgery:

1. Controlled spillage by puncture and drainage of cyst with a trocar [10]

2. Creation of cleavage plane between cyst and ovary, dissection with water, scissors and gravity without traction on cyst [11]

3. Removing cysts in an endobag significantly reduces operating time and spillage as compared to direct retrieval ( $4 \%$ vs. $43.3 \%$ in one study [7] and $13.6 \%$ vs. $62 \%$ in another [9])

4. Controlled intra-peritoneal spillage does not increase post-operative morbidity as long as the peritoneal cavity is thoroughly washed [7]. An experimental study has proved that saline lavage decreases inflammation and adhesion formation to control levels [4]

5. Vaginal removal of dermoid cyst also decreases spillage as compared to laparoscopic retrieval [12]

There is very limited information in published literature on the management of chemical peritonitis following rupture of dermoid cyst contents. The use of systemic anti-inflammatory agents has been described [13]. Most cases are managed conservatively. Cases with more serious complications need multidisciplinary input.

\section{Conclusion}

This case will serve as a reminder for surgeons to exercise extra caution and use spillage preventive strategies when operating on large dermoid cysts. Patients who endure complications often request a medico-legal opinion and frequently the problem originates from insufficient preoperative information. This case report will help to increase awareness of rare but potential complications of iatrogenic spillage of dermoid cyst contents. 


\section{References}

1. Medeiros LR, Fachel JMG et al (2005) Laparoscopy versus laparotomy for benign ovarian tumours. Cochrane Database of Systemic Reviews, Issue 3.Art.No.:CD004751

2. Mecke H, Sayyas V (2001) Laparoscopic surgery of dermoid cysts - intraoperative spillage and complications. Eur J Obstet Gynecol Reprod Biol 96(1):80-84

3. Milingos S, Protopapas A et al (2004) Laparoscopic treatment of ovarian dermoid cysts: eleven years experience. J Am Assoc Gynecol Laparosc 11(4):478-485, Nov

4. Fielder EP, Guzick DS et al (1996) Adhesion formation from release of dermoid contents in the peritoneal cavity and effect of copious lavage: a prospective, randomized, blinded, controlled study in a rabbit model. Fertil Steril 65(4):852-859

5. Zanetta G, Ferrari L et al (1999) Laparoscopic excision of ovarian dermoid cysts with controlled intraoperative spillage. Safety and efficacy. J Reprod Med 44(9):815-820

6. Yuen PM, Yu KM et al (1997) A randomized prospective study of laparoscopy and laparotomy in the management of benign ovarian masses. Am J Obstet Gynecol 177:109-114
7. Campo S, Garcea N (1998) Laparoscopic conservative excision of dermoid cysts with and without an endobag. J Am Assoc Gynecol Laparosc 5(2):165-170

8. Berg C, Berndorff U et al (2002) Laparoscopic management of ovarian dermoid cysts. A series of 83 cases. Arch Gynecol Obstet 266(3):126-129

9. Nezhat CR, Kalyoncu S et al (1999) Laparoscopic management of ovarian dermoid cysts: ten years experience. JSLS 3:179-184

10. Achtari C, Genolet PM et al (1998) Chemical peritonitis after iatrogenic rupture of a dermoid cyst of the ovary treated by coelioscopy. Apropos of a case and review of the literature. Gynakol Geburtshilfliche Rundsch 38(3):146-150

11. Remorgida V, Magnasco A et al (1998) Four year experience in laparoscopic dissection of intact ovarian dermoid cysts. J Am Coll Surg 187(5):519-521

12. Ferrari MM, Mezzopane R et al (2003) Surgical treatment of ovarian dermoid cysts: a comparison between laparoscopic and vaginal removal. Eur J Obstet Gynecol Reprod Biol 109 (1):88-91

13. Rubod C, Triboulet JP et al (2007) Ovarian dermoid cyst complicated by chemical peritonitis. A case report. Gynecol Obstet Fertil 35(7-8):651-653 